\title{
Supercritical string cosmology drains the swampland
}

\author{
John Ellis $\oplus^{1,2}$ Nick E. Mavromatos ${ }^{1},{ }^{1}$ and D. V. Nanopoulos $\oplus^{3}$ \\ ${ }^{1}$ Theoretical Particle Physics and Cosmology Group, Physics Department, King's College London, \\ Strand, London WC2R 2LS, United Kingdom \\ ${ }^{2}$ Theoretical Physics Department, European Organization for Nuclear Research (CERN), \\ CH-1211 Genève 23, Switzerland \\ and National Institute of Chemical Physics \& Biophysics, Rävala 10, 10143 Tallinn, Estonia \\ ${ }^{3}$ George P. and Cynthia W. Mitchell Institute for Fundamental Physics and Astronomy, \\ Texas A\&M University, College Station, Texas 77843, USA, Astroparticle Physics Group, \\ Houston Advanced Research Center (HARC), Mitchell Campus, Woodlands, Texas 77381, USA \\ and Academy of Athens, Division of Natural Sciences, Athens 10679, Greece
}

(Received 19 June 2020; accepted 7 August 2020; published 26 August 2020)

\begin{abstract}
The first and second swampland conjectures (FSC \& SSC) are substantially modified in noncritical string cosmology, in which cosmic time is identified with the timelike Liouville mode of the supercritical string. In this scenario the Friedmann equation receives additional contributions due to the noncriticality of the string. These are potentially important when one seeks to apply the Bousso bound for the entropy of states that may become light as the dilaton takes on trans-Planckian values, as in a de Sitter phase, and restore consistency with the FSC and in at least some cases also the SSC. The weak gravity conjecture (WGC) for scalar potentials is saturated in the supercritical string scenarios discussed in this work, but only if one uses the dilaton as appears in the string effective action, with a kinetic term that is not canonically normalized. In the case of a noncritical Starobinsky potential, the WGC is satisfied by both the canonically normalized dilaton and the dilaton used in the string effective action.
\end{abstract}

DOI: 10.1103/PhysRevD.102.046015

\section{INTRODUCTION}

One of the outstanding challenges for string theory is to identify experimental measurements that could test some characteristic prediction derived from the theory. Confronted with the tremendous gap between the primary formulation of string theory in multiple dimensions at a distance scale beyond the direct reach of observation, the conventional approach to this experimental challenge has been to formulate and probe some effective field theory that describes four-dimensional physics at accessible energy scales that is derived from (or at least motivated by) string theory. Unfortunately, this approach has long been stymied by the enormous ambiguity in the choice of possible effective field theory.

A promising way forward has been provided by the swampland conjectures [1-5], which postulate some very general properties of the possible effective field theory, whose violation would lead to the conclusion that (our

Published by the American Physical Society under the terms of the Creative Commons Attribution 4.0 International license. Further distribution of this work must maintain attribution to the author(s) and the published article's title, journal citation, and DOI. Funded by SCOAP . current understanding of) string theory does not describe Nature. This line of attack is particularly intriguing because some aspects of the swampland conjectures appear to be in tension with the appearance of a cosmological constant in present-day cosmology as well as the commonly studied models of inflation in the early Universe, and may raise questions about the formulation of spontaneous symmetry breaking in the Standard Model of particle physics [6].

One possible way to resolve these issues may be to abandon the conventional effective field theory description of physics in the infra-red limit at energies far below the putative string scale. Effective field theory emerges naturally from critical string theory via its formulation in terms of theory on the string world sheet. We have long argued that conformal field theory is just one aspect of the underlying string theory, and that critical string theory should be regarded as embedded in a broader framework that includes supercritical string scenarios in which there is a timelike Liouville mode that can be identified with cosmic time in scenarios for an evolving Universe [7-11].

As we have discussed previously, the nontrivial dynamics of such a Liouville mode modifies the equations of motion of a conventional effective field theory. In particular, as we emphasize in this paper, in the cosmological context the supercritical dynamics introduces extra terms into the Friedmann equation describing the evolution of the 
Universe, reopening the possibility of a stringy description of inflation in the past and/or dark energy today.

We recall in this context that an essential feature of perturbative critical string theory is the existence of a perturbative S-matrix. This requirement appears to exclude a de Sitter background, since it does not accommodate asymptotic states because of the presence of a horizon [12]. On the other hand $[8,11,13]$, supercritical strings are formulated without requiring the existence of an S-matrix [13], and hence can accommodate both an early near-de Sitter epoch from which the Universe exits smoothly and another near-de Sitter epoch at late times. ${ }^{1}$

The outline of this paper is as follows. In Sec. II we outline relevant aspects of the swampland conjectures (SC). Then, in Sec. III we examine the swampland from the point of view of noncritical string theory. Section IV provides a parallel discussion of the weak gravity conjecture (WGC) in the framework of noncritical string theory.

\section{THE SWAMPLAND CONJECTURES}

There are several swampland conjectures (SC) that bear upon the possible embedding of a de Sitter (dS) solution in a low-energy effective field theory arising from a microscopic string theory model that is supposed to provide a self consistent UV completion.

(i) The distance in field space conjecture (DFSC) [1]

When considering a scalar field $\phi$ within the effective low-energy field theory (EFT) of some string theory, which is displaced by a "distance" $\Delta \phi$ in field space from some initial value, the validity of the EFT is guaranteed provided

$$
\kappa|\Delta \phi| \lesssim c_{1}>0,
$$

where $c_{1}$ is a positive constant of order $\mathcal{O}(1)$, and $\kappa=\frac{1}{M_{\mathrm{Pl}}}$ is the gravitational constant in four space-time dimensions, where $M_{\mathrm{Pl}}=2.4 \times 10^{18} \mathrm{GeV}$ is the reduced Planck mass. In general, when (1) is violated towers of string states descend from the UV, becoming light and thus "contaminating" the EFT, jeopardizing any physical conclusions that may be derived from it.

(ii) The first swampland conjecture (FSC) [2]

This conjecture restricts the self-interactions of scalar field(s) in an EFT stemming from string theory via the gradient of the scalar potential $V$ in field space, so that the EFT is valid:

$$
\frac{|\nabla V|}{V} \gtrsim c_{2} \kappa>0
$$

\footnotetext{
${ }^{1}$ In some supercritical string cosmologies [11], the Universe approaches at large cosmic times a linearly expanding universe [14] that is characterized by a logarithmic dependence of the dilaton on cosmic time, in which case the perturbative S-matrix can be well-defined asymptotically [13]
}

where $c_{2}$ is a positive (dimensionless) constant of $\mathcal{O}(1)$. The gradient in field space is in the multicomponent space of scalar fields $\phi_{i}, i=1, \ldots N$ that the EFT contains.

The constraint (2) can be derived from the DFSC (1), using entropy in de Sitter space [3], by using the Bousso bound [15], which is in this case saturated by the GibbonsHawking entropy [16]. Specifically, let us consider an EFT of a quintessence field $\phi(t)$ in which, say, the latter increases with cosmic time, i.e., $\phi(t)$ has $\dot{\phi}>0$. $^{2}$ As the time evolves, if the field lies in regions in which (2) is violated, towers of string states with masses $m \sim \exp (-a|\Delta \phi|)$, where $a>0$ is a constant with mass dimension -1 that depends on the details of the microscopic string theory, become lighter and lighter and descend from the UV-complete theory to contaminate the EFT as the distance $\Delta \phi$ from the initial point increases.

The number of these effective light degrees of freedom $N(\phi)$ depends on the value of the field $\phi$ at any moment in time, and can be parametrized as [2]:

$$
N(\phi)=n(\phi) \exp (b \kappa \phi),
$$

where $n(\phi)$ indicates the number of states in the tower that are becoming light, and $b$, like $a$, is another positive constant that depends on the mass gap and other details of the underlying string theory. Since, according to the distance conjecture, more states become light as $\phi$ grows, one has to take the (positive) function $n(\phi)>0$ to be monotonically increasing with $\phi$, i.e., $\frac{d n(\phi)}{d \phi}>0$.

In an accelerating Universe, characterized by a Hubble parameter $H$, which for (near-)de Sitter-times is (approximately) constant, the entropy of this tower of string states increases with the Hubble horizon $1 / H$ as

$$
S_{\text {string states }}\left(N, H^{-1}\right)=N^{\gamma}(\kappa H)^{-\delta},
$$

where $\gamma, \delta>0$. On the basis of string examples, the authors of [3] argued that $0<\delta \leq 2$. If the tower of string states that are becoming light behave effectively as point particles, then $\delta=0$, but this is not necessarily the case, and the value of $\delta$ depends on the underlying microscopic string theory.

Since the expanding Universe is characterized by the presence of the Hubble horizon of area $A=4 \pi H^{-2}$, the entropy is bounded by Bousso's covariant entropy constraint applied to a cosmological background [15]: $S_{\text {string states }}\left(N, H^{-1}\right) \leq \frac{1}{4} A$, which is nothing other than the Gibbons-Hawking entropy [16]:

$$
N(\phi)^{\gamma}(\kappa H)^{-\delta} \leq 8 \pi^{2}(\kappa H)^{-2},
$$

from which we derive

\footnotetext{
${ }^{2}$ This is a convention for the definition of the flow, which is made for concreteness, see the explicit examples below.
} 


$$
H^{2} \leq \frac{1}{\kappa^{2}}\left(\frac{8 \pi^{2}}{N(\phi)^{\gamma}}\right)^{2 /(2-\delta)}
$$

If the potential energy of the scalar field dominates the energy density of the Universe, and one assumes the standard Friedmann equation

$$
H^{2} \simeq \frac{\kappa^{2}}{3} V
$$

i.e., the kinetic energy of the scalar field responsible for inflation is ignored, compared to the potential energy, then we can use (7) to rewrite (6) as

$$
\frac{\kappa^{4} V}{3} \leq\left(\frac{8 \pi^{2}}{N(\phi)^{\gamma}}\right)^{2 /(2-\delta)}
$$

After some straightforward manipulations, and taking into account the fact that the derivative of the potential with respect to the field $\phi$ is negative, ${ }^{3}$ we find that

$$
\frac{\left|V^{\prime}\right|}{V} \geq \frac{2}{2-\delta}\left(\ln \left[N^{\gamma}\right]\right)^{\prime},
$$

where the prime denotes the derivative with respect to the field $\phi$. Using (3), one then obtains

$$
\frac{\left|V^{\prime}\right|}{V} \geq \frac{2 \gamma}{2-\delta}\left(\frac{n^{\prime}}{n}+b \kappa\right)>\frac{2 b \gamma}{2-\delta} \kappa
$$

where the last inequality follows from the fact that $n^{\prime}>0$. Comparing (9) with (2), we obtain the FSC with $c_{2}=\frac{2 b \gamma}{2-\delta}>0$, provided that the parameters $b, \gamma$ and $\delta$ are such that $c_{2}$ is of order $O(1){ }^{4}$

The FSC (2) rules out slow-roll inflation, since it implies that the parameter $\epsilon=(\sqrt{2} \kappa)^{-1}\left(\left|V^{\prime}\right| / V\right)$ is of order one, in conflict with phenomenologically successful inflationary models [18].

In fact, it was argued in $[3,4]$ that the FSC does not necessarily hold, but that consistency of the EFT requires either the constraint (2), or:

(iii) The second swampland conjecture (SSC) $[3,4]$

According to the SSC, the minimum eigenvalue of the Hessian in theory space $\min \left(\nabla_{i} \nabla_{j} V\right)$, with the notation $\nabla_{i}$ denoting the gradient of the potential $V$ with respect to the scalar field $\phi_{i}$, should satisfy the following constraint near the local maximum of the potential $V$, provided there is one:

$$
\frac{\min \left(\nabla_{i} \nabla_{j} V\right)}{V} \leq-c_{3} \kappa^{2}<0
$$

\footnotetext{
${ }^{3}$ Here we adopt the conventions of, e.g., the work in [17], as we are interested in the flow of the field from large values, where the de Sitter phase occurs, to small values, where the exit from inflation takes place.

${ }^{4}$ As already remarked, $\delta=0$ if only pointlike states are included in the tower of states $N(\phi)$.
}

where $c_{3}>0$ is an appropriate positive (dimensionless) constant that is $\mathcal{O}(1)$.

We note that, for single-field inflationary models, the condition (11) would also be incompatible with the smallness in magnitude of the second of the slow-roll parameters $\eta$, as required for conventional slow-roll inflationary models.

We also remark that in case of models for which the SSC applies but not the FSC, the entropy-bound-based derivation of FSC still holds, but for a range of fields further away from the regime for which the local maximum of the potential occurs (as required for the implementation of the SSC). The critical value (range) of the field magnitude for the entropy-bound implementation of the FSC depends on the underlying microscopic model, upon which the parameters $\gamma, b$ in the bound (10) also depend.

\section{SUPERCRITICAL STRING THEORY AND THE SWAMPLAND}

The central point of our discussion is the modification of the first swampland conjecture (FSC) (2) in supercritical string theory, in which the right-hand-side (rhs) of the Friedmann equation of motion (7) receives additional contributions due to noncritical string degrees of freedom $[8,9]$, which arise from identifying time as the Liouville mode of the supercritical string [10]. To see this, we consider a concrete but representative example of a supercritical string cosmology where the Liouville mode is identified with cosmic time, and the dilaton plays the role of a quintessence field. The scenario is discussed in detail in [9], and here we simply outline the main results relevant for our purposes.

\section{A. The supercritical string cosmology approach}

Before going into calculational details, it is important to recall briefly the underlying philosophy of the noncritical approach to string cosmology of [8]. This necessarily involves infinite towers of stringy states that fail to decouple from the light degrees of freedom. In the approach of [8] these string states provide a nontrivial environment for the low-energy effective field theory (EFT), whose description incorporates only local field theory modes. When the string is noncritical, the world sheet Weylanomaly coefficients, which appear as the renormalizationgroup $\beta$-functions of the couplings/target-space fields $g^{i}$ of the $\sigma$-model that describes string propagation, are no longer zero as in a conformal field theory corresponding to a critical string model. Instead, they are determined by a standard Liouville-dressing procedure ${ }^{5}$ :

$$
g^{i \prime \prime}+Q g^{\prime}=-\beta^{i}(g) \neq 0
$$

\footnotetext{
${ }^{5}$ For notation, conventions and more details we refer the reader to [8] and references therein.
} 
where the $g^{i}$ are Liouville-dressed couplings/background fields in the $\sigma$-model, which has $d+1$ coordinates, of which $d$ are spacelike coordinates in target space, and one is timelike and interpreted as the target time. The prime in (12) denotes differentiation with respect to the world sheet zero-mode $\rho$ of the Liouville field $\rho(\sigma)$, where $\sigma$ denotes collectively the dependence on world sheet coordinates, which is identified with the target time in the approach of [8]. The Liouville mode itself is viewed as a dynamical renormalization-group scale, which is local on the world sheet and promoted to a fully fledged quantum field on the two-dimensional world sheet geometry. The right-hand side of (12) vanishes in critical string theories, and this absence provides the standard target-space equations of motion of local fields in a low-energy string EFT. The identification with the target time of this extra coordinate in the target space of the string, which in supercritical strings has a timelike signature, imposes a constraint on the $(d+2)$-dimensional target manifold, such that there is a single physical time.

A prototype that illustrates how towers of string states may affect the Weyl-invariance conditions and leading to Liouville dressing is the cigar-type black-hole background in two-dimensional string theory [19], which may be embedded in higher dimensions [20]. As observed in [7], such a stringy background leads to the mixing with light modes of infinite towers of topological modes of (nonpropagating) massive string states with discrete momenta. If they are not taken into account, the truncated $\sigma$-model that includes only backgrounds from the massless string states (the graviton and dilaton in this example) is no longer conformally invariant, rendering the string noncritical. Liouville dressing is then required to restore world sheet conformal invariance, and thus independence of the target-space physics from world sheet dynamics. ${ }^{6}$

Other examples of such mixing between massive and massless states occur in D-brane theories [17,21], when one takes into account the space-time distortions induced by the recoil of such extended objects during scattering with string states representing low-energy matter. Such an approach can also lead to a time-dependent vacuum energy, with a dark energylike equation of state that exhibits temporal dependence (relaxation) [22]. ${ }^{7}$ This cosmological situation

\footnotetext{
${ }^{6}$ Studies of such stringy black holes, and the mixing of the environment of higher string modes, prompted the authors of [7] to discuss quantum coherence issues and modifications of naive quantum mechanics within such an EFT framework, identifying the Liouville mode with time and mapping the problem to a suitable supercritical string.

${ }^{7}$ For phenomenological consequences of relaxation models for the cosmological dark energy in the context of noncritical string cosmologies of [14], see [23]. We also note that supercritical string cosmologies of the type we focus our attention upon here [9] could also contribute to the alleviation of the apparent $H_{0}$ tension [24].
}

is similar in spirit to the effective field theory model of a running vacuum [25], but involves brany objects whose recoil induces the temporal dependence via noncriticality of the underlying string theory. ${ }^{8}$

When applied to specific noncritical string models $[8,9,11]$, the Eq. (12) result in a coupled system of equations that constitute an extension of the cosmological equations of standard critical string cosmologies. The simplest and most representative systems we deal with here, which suffice for our purposes, contain dilaton and metric backgrounds only. In more realistic string models [11] one considers other fields, such as fluxes, string moduli, antisymmetric tensors, etc., which provide additional equations of motion and constraints among the relevant parameters of the models, but do not affect our basic conclusions on the fate of the first swampland conjecture (2) in the noncritical cosmology framework.

We conclude this summary by commenting on the relation between the approach adopted here and the linear-dilaton string cosmology of [14]. The latter is regarded as a critical, "equilibrium" string configuration, which is described by a conformal field theory on the world sheet, even though the internal central charge is noncritical. In the approach of [8], the presence of the Liouville mode and its identification with target time provides a nonequilibrium "transient" period for the associated string universe, which may in some instances tend asymptotically $[9,11]$ to the equilibrium critical string model of [14].

\section{B. A representative supercritical string cosmology model and the swampland conjectures}

Considering metric and dilaton cosmological backgrounds, along with some generic matter and radiation (denoted by the suffix $m$ ), one finds the following cosmological equations in the Einstein frame, upon identifying the (supercritical) Liouville mode with the target time?

$$
\begin{aligned}
3 H^{2}-\tilde{\varrho}_{m}-\varrho_{\phi} & =\frac{e^{-\sqrt{2} \phi}}{2} \tilde{\mathcal{G}}_{\phi}, \\
2 \dot{H}+\tilde{\varrho}_{m}+\varrho_{\phi}+\tilde{p}_{m}+p_{\phi} & =\frac{\tilde{\mathcal{G}}_{i i}}{a^{2}}, \\
\ddot{\phi}+3 H \dot{\phi}+\frac{\partial V_{\text {all }}(\phi)}{\partial \phi}-\frac{1}{\sqrt{2}}\left(\tilde{\varrho}_{m}-3 \tilde{p}_{m}\right) & =+\frac{3}{2} \frac{\tilde{\mathcal{G}}_{i i}}{a^{2}} \\
& +\frac{e^{-\sqrt{2} \phi}}{2} \tilde{\mathcal{G}}_{\phi},
\end{aligned}
$$

\footnotetext{
${ }^{8} \mathrm{An}$ embedding of the running vacuum model in critical string theory and its cosmological implications have been discussed in [26].

${ }^{9}$ For notational convenience in the rest of the article, unless otherwise stated, we work in units with $\kappa=1$.
} 
where $\tilde{\varrho}_{m}$ and $\tilde{p}_{m}$ are the conventional matter density and pressure, $\varrho_{\phi}$ and $p_{\phi}$ are the dilaton energy density and pressure, and ${ }^{10}$ :

$$
\begin{aligned}
\tilde{\mathcal{G}}_{\phi}= & -e^{\sqrt{2} \phi}\left(\frac{1}{\sqrt{2}} \ddot{\phi}+\frac{1}{2} \dot{\phi}^{2}+\frac{Q(t)}{\sqrt{2}} e^{-\phi / \sqrt{2}} \dot{\phi}\right), \\
\tilde{\mathcal{G}}_{i i}= & 2 a^{2}\left(-\frac{1}{\sqrt{2}} \ddot{\phi}-\frac{3}{\sqrt{2}} H \dot{\phi}+\frac{1}{2} \dot{\phi}^{2}+(1-q) H^{2}\right. \\
& \left.-\frac{1}{\sqrt{2}} Q(t) e^{-\phi / \sqrt{2}}(\dot{\phi}-\sqrt{2} H)\right),
\end{aligned}
$$

where $Q^{2}(t)$ is the central-charge surplus, $a$ the cosmic scale factor and $q$ the cosmic deceleration, and we note that only two of the equation (13) are independent. We have used in the above a dilaton field with a canonically normalized kinetic term, following the notation in [17]. In particular, the dilaton $\phi$ used in [17] and appearing in (13) is related to the standard dilaton $\Phi$ used in [14] and [8,9] by

$$
\phi=-\sqrt{2} \Phi .
$$

In our conventions, the slope of the potential is positive, as the potential increases for increasing $\phi$, until it assumes an approximately constant value for large values of $\phi$.

In the convention (15), the string coupling is given by

$$
g_{s}=g_{s}^{(0)} e^{\Phi}=g_{s}^{(0)} e^{-\phi / \sqrt{2}},
$$

and, as we discuss below, in all our noncritical cosmology examples $[8,9,14,17]$, the string coupling is weak, including during the inflationary era, in the sense that the dilaton takes on large (trans-Planckian) positive values. Indeed it is an increasing function of the cosmic time. One can take $g_{s}^{(0)}<1$ to guarantee string-loop perturbation theory in all the phenomenologically relevant epochs of the Universe evolution, from early cosmology to the current era.

The dots above a quantity in (13) denote derivatives with respect to the Einstein-frame cosmic time, which is the standard time used in cosmology to compare models with observations [18]. The rhs of these equations are provided by the terms that are associated with the noncritical string behavior. The time derivatives appearing on the rhs are essentially derivatives with respect to the Liouville mode, which is the zero mode of the world sheet renormalization scale and has been constrained to be identical to the cosmic time [8]. Such terms are absent in critical string cosmologies, including the asymptotic situation met in [14].

We ignore for our purposes here the matter/radiation contributions denoted by the suffix $m$, as we are only interested in early (inflationary) epochs, in particular the validity of the swampland conjectures for de Sitter space

\footnotetext{
${ }^{10}$ We note for completeness [9] that the function $\tilde{\mathcal{G}}_{00}$, which is the 00 component of $\tilde{\mathcal{G}}_{\mu \nu}$, vanishes because the corresponding component of the metric is constant.
}

time and the EFT in the context of noncritical string cosmologies, and hence set $\tilde{\varrho}_{m}=\tilde{p}_{m}=0$.

The reader should notice the dissipative terms proportional to $Q(t) \dot{\phi}$ in (14), where $Q(t)$ is the central charge surplus, that depends in general on the cosmic time [9]. There are many explicit ways to generate such as a surplus, and we shall come back to explicit examples discussed below. In general, the variation of the central charge deficit $Q(t)$ with the cosmic time is provided $[9,11]$ by the CurciPaffuti equation [27], which expresses the renormalizability of the world sheet theory. To leading order in an $\alpha^{\prime}$ expansion, to which we restrict ourselves in this work, this equation is given by

$$
\frac{d \tilde{\mathcal{G}}_{\phi}}{d t}=-6 e^{\sqrt{2} \phi}\left(H-\frac{1}{\sqrt{2}} \dot{\phi}\right) \frac{\tilde{\mathcal{G}}_{i i}}{a^{2}}
$$

in the Einstein frame.

In Eq. (14) above, $q$ denotes the deceleration parameter of the noncritical string Universe $q \equiv-\ddot{a} a / \dot{a}^{2}$ as function of the time. We shall be interested in (almost) de Sitter space-times for which

$$
q \simeq-1
$$

The potential appearing in (13) above is defined by

$$
V_{\text {all }}(\phi)=\left(Q^{2}(t) \exp \left(-\sqrt{2}\left|c_{0}\right| \phi\right)+V_{0}\right),
$$

where $c_{0}$ is a constant to be determined by consistency with the modified swampland criteria, and for generality we have allowed for a potential term in the string action $-\sqrt{-G} V_{0}$ in addition to that dependent on the central charge deficit term. We expect that $V_{0}$ might be generated by, e.g., string-loop effects, or else by interaction with D-particle defects [17], which themselves provide a source of noncriticality. In our convention, inflation is obtained for large positive values of the field $\phi$, which correspond to early cosmic times.

The form of the potential (19) is indicated in Fig. 1. The $Q$-dependent part of the potential is typical of noncritical string cosmologies, and is associated with the centralcharge surplus of the underlying world sheet Liouville theory [8,14]. Indeed, for constant $Q$ (as, e.g., in the case of [14]) and $V_{0}=0$ the potential exhibits a standard quintessencelike behavior, which is compatible with the first swampland conjecture (FSC) (2), provided $c_{0}$ is of order one. For instance, the case of a linearly expanding universe with four uncompactified target-space dimensions studied in [14] has $c_{0}=1$, and thus falls in this category.

The problems with the FSC start when one considers a large-field de Sitter phase (no matter how brief) within the EFT framework, for which the dilaton $\phi>1 / \kappa, V>0$ but also, as we shall discuss [9], $Q(t)$ is no longer constant. In fact, the purpose of this paper is to discuss the swampland criteria for noncritical string potentials of the form depicted 


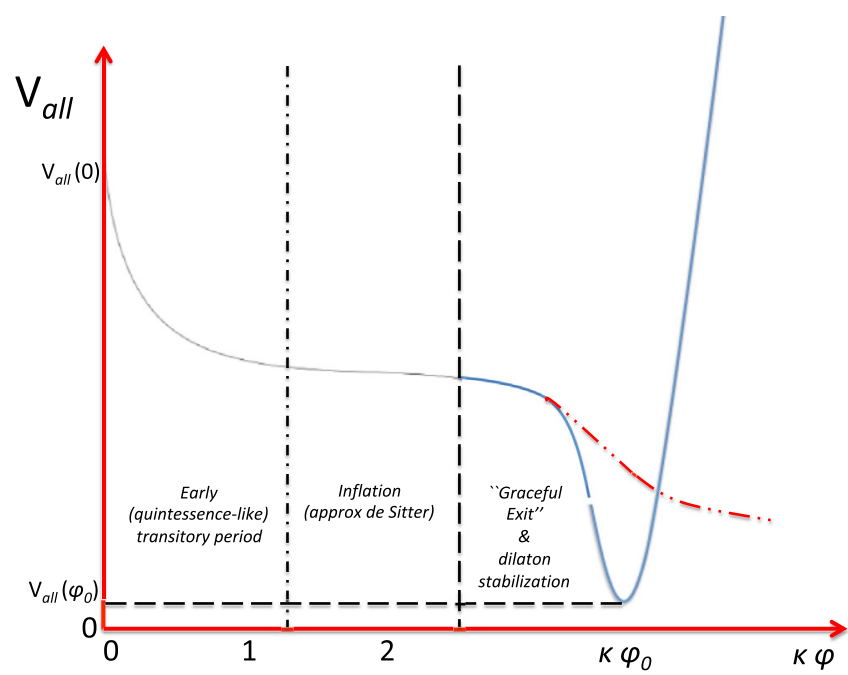

FIG. 1. A typical noncritical string cosmology potential in the range $0<\phi<\infty$. The Universe starts with a quintessencelike behavior for small positive dilaton values, and then passes through an approximately de Sitter era for larger values of $\kappa \phi=\mathcal{O}(1)$, before exiting into an era where the dilaton is stabilized with a value $\phi_{0} \gg 1$. The exit phase (blue curve, to the right of the dashed vertical line) can be characterized by a dilaton-induced Starobinsky-type potential as in the scenario of [17]. In other noncritical string scenarios [11], the dilaton evolves in such a way that the potential exhibits a decaying behavior, $V_{\text {all }} \sim e^{-\tilde{c}_{1} \phi}$ with $\tilde{c}_{1}>0$, at very large times (indicated by the doubly dotted-dashed red curve), and reaches asymptotically a logarithmic behavior corresponding to the linearly expanding Universe of [14], which constitutes an asymptotic "equilibrium" situation for noncritical string cosmology $[8,9,11]$.

in Fig. 1. Depending on the details of the $V_{0}$ part of the potential, the Universe may enter a (relatively brief) de Sitter era, before exiting into either (i) an era in which the dilaton is stabilized at a constant value $\phi_{0}$, as in dilatoninduced Starobinsky-like inflation in the context of the noncritical string/brane scenario of [17] (with the value of the potential $V_{\text {all }}\left(\phi_{0}\right)$ at its minimum being either zero, or small and positive so as to match the current cosmological constant value), or (ii) an era [11] in which the dilaton potential exhibits another noncritical string quintessence form, $V_{\text {all }} \sim e^{-\tilde{c}_{1} \kappa \phi}$, with $\tilde{c}_{1}>0$ some positive constant of $\mathcal{O}(1)$, and the dilaton evolves with the cosmic time as

$$
\phi \propto \ln a,
$$

where $a$ is the scale factor of the Universe. This solution is asymptotic for very large cosmic times to the solution of [14], in which the dilaton scales logarithmically with the Einstein-frame cosmic time, and the Universe is linearly expanding in this frame. ${ }^{11}$

\footnotetext{
${ }^{11}$ It should be noted that, if the expansion is measured in string rods, the linear expansion is not really an expansion of the Universe, but being an asymptotic equilibrium point in the noncritical string evolution [11], this is not an issue.
}

The dilaton energy density and pressure are

$$
\begin{aligned}
\varrho_{\phi} & =\frac{1}{2} \dot{\phi}^{2}+V_{\mathrm{all}}(\phi) . \\
p_{\phi} & =\frac{1}{2} \dot{\phi}^{2}-V_{\mathrm{all}}(\phi) .
\end{aligned}
$$

We observe in (21) that in a de Sitter background of the type we are interested in, under the assumption that the potential dominates, one can ignore the $\dot{\phi}^{2}$ terms in front of $V_{\text {all }}$, so the field $\phi$ acts as a quintessence field inducing an (almost) de Sitter space-time, with

$$
\rho_{\phi} \simeq-p_{\phi} \simeq V_{\text {all }}(\phi) .
$$

The first of Eq. (13) is actually the analogue of the Friedmann equation for noncritical string cosmologies. Using (14) and $\tilde{\varrho}_{m}=\tilde{p}_{m}=0$, we may write it in the following form in the de Sitter phase:

$H^{2} \simeq \frac{1}{3} V_{\text {all }}(\phi)-\frac{1}{3}\left(\frac{1}{2 \sqrt{2}} \ddot{\phi}-\frac{1}{4} \dot{\phi}^{2}+\frac{Q(t)}{2 \sqrt{2}} e^{-\frac{1}{\sqrt{2}} \phi} \dot{\phi}\right)$.

During the de Sitter phase, we may make the reasonable approximation that the behavior (20) characterizes the dilaton field. In particular, we assume the proportionality constant in (20) to be that in the solution of [14], which actually describes the asymptotic exit phase from the noncritical string inflation model of [9]. In such a solution one has:

$$
\dot{\phi} \simeq \sqrt{2} H \quad \Rightarrow \quad \ddot{\phi} \simeq \sqrt{2} \dot{H},
$$

which, on account of the second of the equations (13), implies

$$
\dot{H} \simeq-\frac{3}{2} H^{2} .
$$

From this we easily obtain, using (24):

$$
\frac{\dot{H}}{H^{2}}=\sqrt{2} \frac{H^{\prime}}{H} \simeq-\frac{3}{2} H^{2} \quad \Rightarrow \quad H \simeq H_{I} e^{-\frac{3}{2 \sqrt{2}} \phi},
$$

where the prime denotes differentiation with respect to $\phi$, and $H_{I}$ is an inflationary scale to be determined phenomenologically.

We now observe that, because of (24) and (25), the Curci-Paffuti equation (17) implies that

$$
\tilde{\mathcal{G}}_{\phi}=c_{4},
$$

a constant of mass dimension $[+2]$, during the de Sitter phase, which is realized for large values of $\phi$ [see Fig. 1]. Using (14) and (26), this yields the following evolution with respect to the cosmic time, or equivalently $\phi(t)$, of the central charge deficit $Q(t)$ [9]: 


$$
Q(t) H_{I} \simeq\left(-c_{4}\right) e^{\frac{5}{2 \sqrt{2}} \phi}+\frac{H_{I}^{2}}{2} e^{\frac{3}{2 \sqrt{2}} \phi} .
$$

We now remark that, although the supercriticality of the string corresponds to $Q^{2}(t)>0$, we may without loss of generality assume that $Q(t)>0$, which allows us to take

$$
c_{4}=-\left|c_{4}\right| \leq 0 .
$$

As we see later, this is a self-consistent choice when formulating the modified swampland conditions for supercritical strings.

Then, we obtain from (23)

$$
H^{2} \simeq \frac{4}{7} V_{\text {all }}(\phi)-\frac{6}{7} Q(t) H e^{-\frac{1}{\sqrt{2}} \phi} .
$$

Using the Bousso bound [16], or equivalently (6), which is valid independently of the criticality or otherwise of the string, being a geometric bound on the entropy of states, one may obtain from (24) and (30):

$$
\begin{aligned}
V_{\text {all }}(\phi) & \leq \frac{7}{4}\left(\frac{8 \pi^{2}}{N^{\gamma}}\right)^{2 /(2-\delta)}+\frac{3}{2} Q(t) H e^{-\frac{1}{\sqrt{2}} \phi} \\
& \equiv \frac{7}{4}\left(\frac{8 \pi^{2}}{N^{\gamma}}\right)^{2 /(2-\delta)}+\mathcal{D}_{\phi}(\phi) .
\end{aligned}
$$

where

$$
\mathcal{D}_{\phi}(\phi)=\frac{3}{2}\left|c_{4}\right|+\frac{3 H_{I}^{2}}{4} e^{-\frac{1}{\sqrt{2}} \phi},
$$

as can be inferred from (28) and (29). It is clear that the presence of the function $\mathcal{D}_{\phi}(\phi)$ which depends on the dilaton may drastically modify the swampland bound, depending on the details of the noncritical string model, in particular the values of the parameters $c_{0}$ and $c_{4}$.

Indeed, deep in the de Sitter phase, the field $\phi$ takes on trans-Planckian values, for which the distance conjecture (1) is violated. We may also assume that most of the states that are becoming light behave either in a pointlike way, corresponding to $\delta \simeq 0$, or are stringlike, with $\delta=1$. $^{12}$ Hence, without loss of generality we may assume $0<\delta<$ 2 as a physically relevant range of this parameter for our noncritical string cosmologies, which is also in agreement with a plethora of critical string models [3].

Taking into account the fact that in this large-dilaton regime there is a large number of states (3) that are light, since $n(\phi) \gg 1$, with $b \gamma=\mathcal{O}(1)>0$, our analysis indicates that the term $\mathcal{D}_{\phi}(\phi)$, proportional to $Q(t)$, dominates the $N$-dependent entropy term on the rhs of the inequality

\footnotetext{
${ }^{12}$ Surfacelike states with $\delta=2$ correspond to a singular limit of the $N$-dependent terms on the rhs of (31) and are subleading, carrying zero measure in the space of states.
}

(31). Therefore, using (32) we may approximate the rhs of the inequality in the second line of (31) by

$$
V_{\text {all }}(\phi) \lesssim \frac{3}{2}\left|c_{4}\right|+\frac{3 H_{I}^{2}}{4} e^{-\frac{1}{\sqrt{2}} \phi},
$$

where we stress once again that the (dimensionful) constant $c_{4}$ is connected to the supercritical-string central-charge surplus $Q$, and vanishes for critical strings: $c_{4}=0$.

The potential (19) may be written as

$$
\begin{aligned}
V_{\mathrm{all}}= & c_{4}^{2} e^{\left(5-2\left|c_{0}\right|\right) \frac{1}{\sqrt{2}} \phi}+\frac{H_{I}^{4}}{4} e^{\left(3-2\left|c_{0}\right|\right) \frac{1}{\sqrt{2}} \phi} \\
& +H_{I}^{2}\left|c_{4}\right| e^{\left(4-2\left|c_{0}\right|\right) \frac{1}{\sqrt{2}} \phi}+V_{0} .
\end{aligned}
$$

For consistency with the (quintessencelike) exponential behavior of the potential depicted in Fig. 1, which we take as our prototype of supercritical string cosmology, it suffices to consider potentials (34) for which either (i) all of the exponents of the exponentials are negative, or (ii) the largest of them vanishes, and the other two are negative, i.e.,

$$
\left|c_{0}\right| \geq 5 / 2
$$

Case (i): All the exponents in the exponential are negative

For large positive $\phi$, the leading term in the potential is that with coefficient $c_{4}^{2}$. We then see from (19) and (31) that for consistency with inflationary phenomenology, a sufficient condition for a de Sitter phase at large $\phi$ is

$$
0<V_{0} \sim H_{I}^{2} \lesssim \frac{3}{2}\left|c_{4}\right|
$$

In this way satisfying the second swampland criterion becomes almost trivial. Indeed, taking the logarithms on both sides of (33), differentiating with respect to the field $\phi$, and recalling that in our case $V_{\text {all }}^{\prime}<0$ during and before the inflationary phase [see Fig. 1], we obtain

$$
\frac{\left|V_{\text {all }}^{\prime}\right|}{V_{\text {all }}} \gtrsim \frac{3 H_{I}^{2}}{4} e^{-\frac{1}{\sqrt{2}} \phi} \frac{1}{\frac{3}{2}\left|c_{4}\right|+\frac{3 H_{I}^{2}}{4} e^{-\frac{1}{\sqrt{2}} \phi}}+O\left(n(\phi)^{-\frac{2 \gamma}{2-\delta}} e^{-\frac{2 b \gamma}{2-\delta} \phi}\right) .
$$

which provides a much weaker constraint on the potential than (2) for large $\phi \gg 1$. We reiterate that above we assumed the presence of a large number of light string states, in which case the terms $\mathcal{O}(\ldots)$ on the rhs of the inequality (37) are subleading for $b, \gamma=\mathcal{O}(1)>0$, as explained previously. Otherwise, the standard swampland condition is recovered, which is the case for critical strings.

We now check the conditions on the parameters of the potential (34) under which it satisfies the condition (37) in 
the large- $\phi$ (de Sitter) regime (see Fig. 1). To this end, we compute the quantity:

$\frac{\left|V_{\text {all }}^{\prime}\right|}{V_{\text {all }}} \simeq \frac{|5-2| c_{0}||}{\sqrt{2}} c_{4}^{2} e^{\frac{\left(5-2\left|c_{0}\right|\right)}{\sqrt{2}} \phi} \frac{1}{V_{0}+c_{4}^{2} e^{\frac{\left(5-2\left|c_{0}\right|\right)}{\sqrt{2}} \phi}+\ldots}$,

where the $\ldots$ denote subleading terms in the large-field de Sitter regime $\phi \gg 1$ with $\left|c_{0}\right|>5 / 2$. Comparing (38) with (37), we observe that (37) is satisfied provided:

$$
\begin{aligned}
5-2\left|c_{0}\right| & =-1 \quad \Rightarrow \quad\left|c_{0}\right|=3, \quad \text { and } \\
\frac{c_{4}^{2}}{V_{0}} & \gtrsim \frac{1}{\sqrt{2}} \frac{H_{I}^{2}}{\left|c_{4}\right|},
\end{aligned}
$$

which, since $V_{0} \sim H_{I}^{2}$ (36), implies:

$$
\kappa^{2}\left|c_{4}\right| \gtrsim 0.89\left(H_{I} \kappa\right)^{4 / 3},
$$

where we have reinstated the factors of $\kappa$ units, for clarity. On the other hand, from the Bousso bound on the potential [(36) or equivalently (33)], we must have

$$
\kappa^{2}\left|c_{4}\right| \gtrsim 0.67\left(H_{I} \kappa\right)^{2}
$$

for $\kappa \phi \gg 1$. Taking into account the fact that phenomenologically $H_{I} \kappa \sim 10^{-4} \ll 1$ [18], we find that a stronger lower bound on $\left|c_{4}\right|$ comes from (40), which is the result of the modified swampland condition (37) in the supercritical string model.

Case (ii): One exponent vanishes

Let us now consider the case in which one exponent vanishes, and the other two are negative with

$$
\left|c_{0}\right|=\frac{5}{2} \text {. }
$$

In this case, we see from (19) and (31) that for consistency with inflationary phenomenology, a sufficient condition for obtaining a de Sitter phase at large $\phi$ is

$$
0<\left|c_{4}\right|^{2}+V_{0} \sim \kappa^{-2} H_{I}^{2} \lesssim \frac{3}{2}\left|c_{4}\right| \kappa^{-2},
$$

where we have again reinstated the factors of $\kappa$, for clarity. Performing a similar analysis as before, i.e., by computing $V_{\text {all }}^{\prime} / V_{\text {all }}$ from (34) at large $\phi \gg 1$, but now under the condition (42), we reach the conclusion that in order for $V_{\text {all }}$ to satisfy (37) and (43) simultaneously one must have

$$
\begin{gathered}
\frac{\left|c_{4}\right| H_{I}^{2}}{\left|c_{4}\right|^{2}+V_{0}} \gtrsim \frac{1}{\sqrt{2}} \frac{H_{I}^{2}}{\left|c_{4}\right|} \Rightarrow\left|c_{4}\right|^{2} \gtrsim \frac{\left|c_{4}\right|^{2}+V_{0}}{\sqrt{2}} \sim \frac{H_{I}^{2} \kappa^{-2}}{\sqrt{2}}, \\
\text { and }\left|c_{4}\right|^{2} \gtrsim \frac{4}{9} H_{I}^{4} .
\end{gathered}
$$

The stronger bound on $\left|c_{4}\right|$ is again that coming from the modified swampland constraint (37) on the slope of the dilaton potential.

The condition (37) is necessary and sufficient for the validity of the EFT during the de Sitter phase of supercritical string cosmology. The latter contains a plethora of stringy states, which result in noncriticality, but collectively their presence is taken into account by the presence of the EFT terms on the rhs of (13), stemming from the identification of the Liouville mode with the cosmic time [8]. The modified criterion (33) or equivalently (37) is compatible with slow-roll inflation, in contrast to the first swampland conjecture (2). The latter is recovered in the critical string limit $Q \rightarrow 0$, since the $N$-dependent term on the rhs of the inequality in (31) is the only term present in that case.

\section{Embedding in microscopic conformal field theory models}

The constants $c_{4}$ and $c_{0}$ entering the effective potential (34) would be determined by the embedding of the effective supercritical string cosmology model into a microscopic string realization. For instance, one may consider the framework of [14], in which the internal dimensions correspond to minimal models characterized by specific (discrete) values of their central charges. In such a case, $c_{0}$ and $c_{4}$ are fixed by the requirement that the supercritical string model approaches the linearly expanding Universe model of [14] asymptotically as $\phi \rightarrow \infty$. In this connection, we observe that setting $V_{0}=0$ and

$$
\left|c_{0}\right|=7 / 2
$$

in (34) [which is compatible with (35)], the potential assumes the form

$$
\begin{gathered}
V_{\mathrm{all}}=c_{4}^{2} e^{-\sqrt{2} \phi}+\frac{H_{I}^{4}}{4} e^{-\frac{4}{\sqrt{2}} \phi}+H_{I}^{2}\left|c_{4}\right| e^{-\frac{3}{\sqrt{2}} \phi} \\
\stackrel{\phi \rightarrow \infty}{\Rightarrow} V_{\mathrm{all}}(\phi \rightarrow \infty) \simeq c_{4}^{2} e^{-\sqrt{2} \phi} .
\end{gathered}
$$

The asymptotic form of $V_{\text {all }}$ coincides with the dilaton potential in the model of [14], when we identify $c_{4}^{2}$ with the central-charge surplus $\delta c \propto Q_{\star}^{2}=$ constant.

We recall that, in the approach of [14], if $c_{\text {tot }}$ denotes the total central charge of the world sheet $\sigma$-model theory, and $d$ the number of uncompactified string dimensions, one has [14]

$$
c_{\mathrm{tot}}=d-12 Q_{\star}^{2}+c_{I},
$$

where $c_{I}$ is the central charge corresponding to the conformal field theory of the internal manifold. In the bosonic prototype string theory, decoupling of reparametrization ghosts requires $c_{\text {tot }}=26$ (10 for superstrings), and in our case $d=4$. This implies that 


$$
12 Q_{\star}^{2}=c_{I}-22,
$$

which is a model-dependent constant in the model of [14] that depends on the conformal data of the internal theory, specifically on its central charge $c_{I}$. Supercritical strings correspond to $Q_{\star}^{2}>0 .{ }^{13}$ In terms of the target-space effective action, in the model of [14] there is a positive vacuum energy of quintessence type of the form

$$
\int d^{4} x \sqrt{-g} 4 Q_{\star}^{2} e^{-\sqrt{2} \phi}
$$

in our conventions (15). Comparing (46) with (49), we observe that the value of $c_{4}$ is fixed by the choice of conformal field theory corresponding to the internal manifold degrees of freedom:

$$
c_{4}^{2}=4 Q_{\star}^{2},
$$

where $Q_{\star}^{2}$ is the central charge surplus, ${ }^{14}$ which is a rational real number in the models of [14].

Setting $\phi=0$ in (28) and taking (29) into account, we find

$$
Q(\phi=0)=\frac{\left|c_{4}\right|}{H_{I}}+\frac{1}{2} H_{I},
$$

where $H_{I}$ is expressed in units of $\kappa^{-1}$. For realistic inflationary scenarios [18] $H_{I} \kappa \lesssim 10^{-4} \ll 1$, so in Planck units $H_{I} \ll 1$ in (51), (28), and hence $Q^{2}(0)>Q_{\star}^{2}=Q^{2}(\phi \rightarrow$ $+\infty)$ when (50) is taken into account. One may consider (51) as corresponding to an initial fixed-point conformal field theory(CFT), e.g., another minimal model, following [14], with

$$
Q_{\star 1 \mathrm{CFT}}^{2} \equiv Q^{2}(\phi=0)>Q_{\star}^{2}(\phi \rightarrow \infty) \equiv Q_{\star 2 \mathrm{CFT}}^{2}=\frac{\left|c_{4}\right|}{4} .
$$

In the minimal models considered in [14] as providing conformal models for the internal manifold, $Q_{\star \mathrm{iCFT}}^{2}, i=1$, 2 take discrete rational values.

Thus $Q^{2}(t)$ (28), which defines the overall "running" central charge surplus in the supercritical string model including the contributions from the internal manifold, interpolates (52) between specific minimal models of the type considered in [14]. We note that the evolution (28) of

\footnotetext{
${ }^{13}$ The terminology supercritical refers to the fact that in terms of a string in $D$ flat uncompactified target space time dimensions, $D=d+c_{I}>26$ (or 10 for superstrings).

${ }^{14}$ It corresponds to a Wick-rotated Coulomb charge at infinity for the minimal conformal field theory models considered in [14] as a description of the internal-dimension manifolds of the supercritical string.
}

$Q^{2}(t)$ is independent of the constant $c_{0}$, but assumes implicitly that there exists a slowly running inflationary phase at a scale $H_{I}$. This is not the case in the model of [14], which describes a linearly expanding universe, but it is consistent with the aforementioned role of this theory as an asymptotic fixed point of the supercritical string model [10].

In this approach one considers an interpolating theory with a central-charge surplus $Q^{2}(t)$ dependent on the Liouville mode that is identified with the cosmic time [10], hence there is no overall increase in the target-space dimensionality of the interpolating string model. There is a departure from criticality for the two-dimensional (world sheet) field theory corresponding to the internal manifold of the string. During the interpolating phase, the internal conformal field theory at $\phi=0$, corresponding to an initial cosmic time, is perturbed by relevant operators in a world sheet renormalization-group sense. This induces a flow between the two fixed points described by the conformal field theories corresponding to the Coulomb charges $Q_{\star \mathrm{iCFT}}^{2}, i=1,2$ (52).

During the flow, Liouville dressing is required to maintain $c_{\text {tot }}=26$, as required for ghost decoupling. In this way, the internal central charge $c_{I} \rightarrow c_{I}(t)$ becomes itself a function of the Liouville mode (i.e., cosmic time), so that (48) [or, equivalently, (47)] is satisfied with $c_{\text {tot }}=26$. The theory flows asymptotically to a fixed-point theory corresponding to a dilaton potential of the form (46) for large positive values of $\phi \rightarrow \infty$, where the model asymptotes to one of the conformal field theory models of [14]. In this scenario the constants $c_{4}^{2}(50)$ and $c_{0}$ (45) are determined.

The above serves as an example of how one can determine the relevant constants appearing in the effective dilaton potentials (19) of the supercritical string cosmology models by means of specifying the underlying microscopic world sheet conformal field theory models. As we have emphasized, the models of [14] do not describe, as they stand, inflationary physics. They serve as asymptotic fixed points in a world sheet renormalization-group sense of a more complete theory that describes inflation for relatively small values of the dilaton $\phi>0$, as happens in the model (34) examined above. More complicated supercritical string models also exist in the literature, such as the type-0 string noncritical string theory model of [28] and the tachyon-dilaton models of [29]. Such models are characterized by transient inflationary phases, during which the central charge surplus diminishes as the cosmic time increases as in our case (34) above, before oscillating around zero for a short period of time, and then asymptoting to one of the minimal model values of the linearly expanding Universe of [14].

We stress again that our considerations deal with generic supercritical effective theories, without an emphasis on presenting detailed microscopic string constructions. 
Our approach should be viewed as an attempt to demonstrate that generically, supercritical string with a Liouvillerenormalized central charge surplus $Q^{2}(t)$ can lead to cosmologies that are embeddable in a UV-complete theory of quantum gravity, in which the corresponding EFT bypasses the swampland criteria. The aforementioned specific string models serve as concrete examples of microscopic string theory models where this situation is realized explicitly.

Since our supercritical string (Liouville) cosmology is viewed as an interpolation between critical string models, during the monotonic Liouville evolution taken to correspond in our convention to a dilaton field that increases with the cosmic time, the descent from the UV of massive string states that become massless during this evolution, and thus fail to decouple, is governed by the same criteria as in the critical string cosmologies used in [3] and above when formulating the second swampland conjecture. In particular, the generic parametrization (4) of the entropy of massive string states is valid in our Liouville noncritical string case as well, with the Hubble parameter depending on the timelike Liouville mode that is identified with the cosmic time in our approach [10].

This dependence parametrizes collectively the failure of decoupling of the massive states in the following sense, familiar from our prototype example of a noncritical string theory, realized in the two-dimensional stringy black hole system [7]: (i) one starts from an underlying conformal field theory, corresponding to an initial value of the dilaton field; (ii) then, some specific perturbations, depending on the microscopic content of the theory, move it away from criticality. The perturbations may mix massless and massive states in the spectrum, such that some of the massive stringy states fail to decouple as the dilaton evolves, exactly as happens in the aforementioned black hole example [7]. This failure is encoded in the noncriticality of the string cosmology model that interpolates between critical string backgrounds, and it is in this sense that the effective description of the cosmology in terms of local field theories at low energies, including the dilaton and graviton fields, suffices. Any nonlocality stemming from these massive states is described collectively by the effects of Liouville mode. The embedding of such an EFT into a consistent quantum gravity model is guaranteed by bypassing the swampland criteria, as a consequence of the modified cosmological evolution that characterizes the supercritical string cosmologies [9].

This is the essence of our supercritical string cosmology $[7,10]$ and its evasion of the swampland criteria, as presented above.

\section{The special case of a dilaton-induced Starobinsky-like potential [17]}

In light of the above discussion, we infer that the dilaton/ D-particle-induced Starobinsky-like potential of [17] constitutes a consistent noncritical string cosmology, as it also satisfies (37). However, for completeness we should mention that this model is constructed somewhat differently, in that its dilaton central charge deficit is assumed to be subcritical, as the brane world lives in $d=4$ dimensions, and the noncriticality arises from the interaction with ensembles of D-particles living on the brane world, which enter the latter from the bulk as the brane Universe propagates in cosmic time.

The corresponding effective action in the Einstein-frame is given by [17]

$S_{\text {eff }}^{\text {staro }}=\frac{1}{2 \kappa^{2}} \int d^{4} x \sqrt{-g}\left(R(g)-\left(\partial_{\mu} \phi\right)^{2}-V_{\mathrm{Nc}-\mathrm{Staro}}+\ldots\right)$,

where the four-dimensional gravitational constant is given by $\kappa^{2}=8 \pi \alpha^{\prime} \mathcal{V}_{C}^{-1}$, and $\alpha^{\prime}=M_{s}^{-2}$ is the Regge slope where $M_{s}$ is the string mass scale, and $\mathcal{V}_{C}$ is the volume of the extra compact dimensions (or the volume of the portion of the bulk space between the brane worlds, depending on the construction). The potential $V_{\mathrm{NC}-\text { Staro }}$ is given by [17]

$$
V_{\mathrm{NC}-\mathrm{Staro}}=\mathcal{A}-\frac{44}{3 \kappa^{2} \alpha^{\prime}} e^{-\sqrt{2} \kappa \phi}+\ldots \geq 0 .
$$

The quantity $\mathcal{A}=16 \pi \frac{n \sqrt{\alpha}}{g_{s}^{(0)} \mathcal{V}_{c}}>0$, with $g_{s}^{(0)}<1$, depends on the (three-)density of D-particles on the brane world $n$, which is assumed to be approximately constant, with its dilution due to cosmic expansion being compensated by an influx of D-particles from the bulk. The density $n$ and masses of the D-particles can be adjusted so that the potential vanishes at $\phi=0$. The magnitude of the quantity $A$ provides the inflationary scale in the model.

We now make some important remarks concerning the structure of such a potential. Notice that in the conventions of [17], the potential increases for increasing positive $\phi$, which is a crucial difference from the noncritical string potentials (34). On the other hand, as in the supercriticalstring case, the inflationary phase occurs for large positive $\kappa \phi \gg 1$. Nonetheless, because now $V_{\mathrm{NC}-\text { Staro }}^{\prime}>0$, some crucial modifications need to be made in the noncritical string analysis presented above that led to the swampland conditions.

The potential (54) corresponds to (19), but now $Q^{2}<0$ as in subcritical strings. Formally, the noncritical string analysis can be performed in such a case by analytically continuing to Euclidean cosmic time

$$
t \rightarrow i t, \quad Q \rightarrow i Q, \quad H \rightarrow i H,
$$

maintaining otherwise the structure of (23). Using the analytic continuations of the expressions (25) and (26) and the Curci-Paffuti equation we arrive at a modified evolution equation for the central charge deficit $Q(t)$, which results in 
a modified Bousso bound when we revert to Minkowski cosmic time:

$$
0<V_{\text {NC-Staro }}(\phi) \lesssim \frac{3}{2}\left|c_{4}\right|-\frac{3 H_{I}^{2}}{4} e^{-\frac{1}{\sqrt{2}} \phi},
$$

where we maintain the same sign in front of the constant $c_{4}$ as in the supercritical string case (33), as required by the positivity of the potential (the reader can check from (27) that we have the freedom to do so). However the sign of the $H_{I}^{2}$-dependent term is now the opposite, in view of (55).

Taking the logarithm of (56) and differentiating with respect to $\phi$, we now have

$$
\begin{aligned}
0< & \frac{V_{\text {NC-Staro }}^{\prime}}{V_{\text {NC-Staro }}} \lesssim \frac{3 H_{I}^{2}}{4} e^{-\frac{1}{\sqrt{2}} \phi} \frac{1}{\frac{3}{2}\left|c_{4}\right|-\frac{3 H_{I}^{2}}{4} e^{-\frac{1}{\sqrt{2}} \phi}} \\
& +\mathcal{O}\left(n(\phi)^{-\frac{2 \gamma}{2-\delta}} e^{-\frac{2 b \gamma}{2-\delta} \phi}\right) .
\end{aligned}
$$

From this we see that the condition (37) is now modified by changing the sign of the inequality, since now the slope of the (positive) potential is also positive $V_{\mathrm{NC}-\text { Staro }}^{\prime}>0$.

It is thus straightforward to see that, during the transPlanckian inflationary de Sitter phase with $\kappa \phi \gg 1$, the Starobinsky-like potential (54) satisfies the modified Swampland conjecture (57) quite comfortably. Indeed, we see that

$$
\frac{V_{\text {NC-Staro }}^{\prime}}{V_{\text {NC-Staro }}}=\sqrt{2} \frac{44}{3 \kappa^{2} \alpha^{\prime}} \frac{e^{-\sqrt{2} \kappa \phi}}{\mathcal{A}-\frac{44}{3 \kappa^{2} \alpha^{\prime}} e^{-\sqrt{2} \kappa \phi}+\ldots},
$$

which can be compared with the rhs of the inequality (57). The rhs of (58) is much smaller for phenomenologically relevant values of $H_{I}, \mathcal{A}, \alpha^{\prime},\left|c_{4}\right|=\mathcal{O}(\mathcal{A})$, in view of the difference in the powers in the exponents of the exponentials containing the large $\phi$ field. Thus, the condition (57) is naturally satisfied and an EFT description is valid.

We stress once more that, in our considerations in this work, and more generally in our noncritical cosmological string models $[8,9,11]$, the string coupling remains weak: $g_{s}<1$ during the entire cosmic evolution from the origin of cosmic time, which corresponds to $\phi=0$, until the present era, including the inflationary epoch (see Fig. 1). Hence the D-particles remain heavy in the scenario of [17], since their masses are proportional to

$$
M_{D} \sim g_{s}^{-1}\left(\alpha^{\prime}\right)^{-1 / 2}
$$

and $g_{s}=g_{s}^{(0)} e^{-\frac{2}{\sqrt{2}} \phi}<1$.

Nonetheless, as the dilaton takes on large values, other string states (e.g., scalar moduli or stringy extended objects) can descend from the UV to contaminate the EFT in the way described above. However, in the context of our supercritical strings this is taken into account by representing collectively the "environment" of such states via extra contributions to the EFT cosmological equations, incorporated via the appropriate Liouville dressing and identification of the (timelike) Liouville mode with the cosmic time (see the rhs of (13) in the concrete example discussed in the current work). Thus, we always retain an EFT description, which is reflected in the triviality of the modified swampland condition (37) in supercritical string cosmologies. There are, however, some restrictions on the parameters of the respective dilaton potentials, see, e.g., (35) and (36), needed to match the relative phenomenology.

\section{DISCUSSION: ISSUES WITH THE WEAK GRAVITY CONJECTURE}

Before closing, we discuss the consistency of our results with the weak gravity conjecture (WGC) [30] and its extension to include scalar fields [31]. The WGC states essentially that gravity is the weakest of the fundamental forces in four dimensions. According to the WGC, if a theory contains a U(1) gauge symmetry with coupling $g$, then there must exist a state of charge $q$ and mass $m$, such that [30]

$$
g q M_{P}=m
$$

where $M_{P}=\sqrt{8 \pi} M_{\mathrm{Pl}}$ is the four dimensional Planck scale. Its scalar field extension [31] implies that if $m\left(\phi_{i}\right)$ is the mass gap of some scalar field $\varphi$ (not the dilaton) interacting with the light scalar moduli fields $\phi_{i}$ (which include the dilaton) within some four space-time dimensional EFT, then gravity remains the weakest force, which requires that interactions involving exchanges of the light scalars be such that the mass of the scalar field $\varphi$ satisfies

$$
m^{2} \leq \tilde{g}^{i j} \partial_{i} m \partial_{j} m,
$$

where $\tilde{g}_{i j}$ is the metric in the space of the moduli fields $\phi_{i}$. As we do not deal explicitly with charged matter in this work, it is this scalar extension (61) that is of interest to us here.

First we notice that in models involving (scalar) D-particles, as in the Starobinsky inflation model of [17], the masses of the D-particle states themselves satisfy (61), since that the D-particle mass is inversely proportional to the string coupling (59). In terms of the standard dilaton $\Phi$ (15) that is used in the canonical expression for the D-particle mass, the string coupling is given by $g_{s}=e^{\Phi}$, so we see that condition (61) is satisfied, in fact it is saturated with a constant "metric" in theory space that is equal to the identity. However, having said that, the conjecture is formulated at present for local field theory scalars, and its extension to stringy/brany objects is not yet formulated to a level of precision and understanding that allows us to apply the above result (61) with rigor.

Moreover, in our noncritical string cosmologies, the considerations around the scalar WGC pertain to masses of 
scalars other than the dilaton, which is one of the string moduli. As such, it is not relevant for the noncritical string dilaton potentials (34) discussed in this work. One needs a formulation of the scalar WGC that incorporates the potential of the (self-interacting) dilaton. ${ }^{15}$

In this connection, an improved version of the scalar WGC has been suggested in [32]:

$$
\left(V^{\prime \prime}\right)^{2} \leq\left(2\left(V^{\prime \prime \prime}\right)^{2}-V^{\prime \prime} V^{\prime \prime \prime \prime}\right) \kappa^{-2}
$$

which is expressed in terms of the scalar potential $V$, for canonically normalized fields. This improved WGC resolves the axion puzzle, leading to a plausible constraint on the axion decay constant, $f_{a} \leq M_{P}$, as expected. In principle, (62) should be applicable to our case, where we deal with a canonically normalized dilaton $\phi(15)$.

As discussed in Sec. II, in both the cases (42) and (39) that are compatible with the noncritical string modification of the first swampland conjecture (37), we are dealing with large dilaton field values $\kappa \phi \gg 1$ and exponential potentials in a de Sitter phase of the Universe:

$$
V \sim A e^{\gamma \kappa \phi},
$$

where $\gamma$ is a negative dimensionless constant that takes the value

$$
\gamma=-\frac{1}{\sqrt{2}}
$$

in both cases. For such potentials, the condition (62) is not satisfied.

We remark, though, that there is no rigorous diagrammatic derivation of the improved scalar WGC (62). In particular, the coefficients of the various scalar self-interaction terms appearing on the rhs of the inequality could in principle be modified. For this reason, a generalized form of the scalar potential WGC (62) has been proposed in [33]:

$$
\left(V^{\prime \prime}\right)^{2} \leq\left(2 c_{1}^{\prime}\left(V^{\prime \prime \prime}\right)^{2}-c_{2}^{\prime} V^{\prime \prime} V^{\prime \prime \prime \prime}\right) \kappa^{-2},
$$

where $c_{1}^{\prime}$ and $c_{2}^{\prime}>0$ are positive coefficients of order $\mathcal{O}(1)$. With appropriate values of these coefficients, the generalized form of WGC (65) can accommodate various scalar potentials of interest in the early Universe, as in Q-ball models, but also exponential scalar potentials of the form (63) for $\gamma \leq 1$, such as our supercitical string case with a canonically normalized dilaton (64). The condition would be saturated in that case for $c_{1}^{\prime}=c_{2}^{\prime}=2$.

On the other hand, we note that the condition is satisfied, indeed saturated, if one uses the standard dilaton $\Phi$ (15) appearing in the definition of the string coupling, $g_{s}=e^{\Phi}$,

\footnotetext{
${ }^{15}$ In passing, we mention that the scalar WGC (61) appears to be inconsistent with the postulated properties of axion potentials.
}

as the string loop-counting parameter. However, we note that in such a formalism the dilaton does not have canonically normalized kinetic terms, but appears in the string-inspired low-energy gravitational action in a D-dimensional uncompactified target space in the form:

$$
\begin{aligned}
S_{\text {eff }}= & \frac{1}{2 \kappa^{2}} \int d^{D} x \sqrt{-g} \\
& \times\left(R(g)-\frac{4}{D-2}\left(\partial_{\mu} \Phi\right)^{2}-e^{\frac{4 \Phi}{D-2}} Q^{2}+\ldots\right),
\end{aligned}
$$

where $Q^{2}$ is the central charge surplus of the supercritical string $[8,14]$, and the $\ldots$ denote other fields, higher derivative terms, etc.. Thus, for four large uncompactified dimensions, $D=4$, one obtains the result (15) for the canonically normalized dilaton, $\phi$, used in [17] and above.

The satisfaction of the improved WGC (62) by $\Phi$ but not $\phi$ is curious, but may be the correct form to be used in the case of noncritical strings with exponential scalar potentials. We remark that in [32] the conjecture (62) was derived by looking at four-point scalar interactions, one with the scalar field as a mediator and the second a four-scalar contact interaction, which could be due to the exchange of a massive mediator. The four-point interaction is repulsive, and is associated with the term in the conjecture involving the fourth derivative of the field. Thus, subtracting the contact interaction from the four-point amplitude with a scalar mediator (which is attractive, and corresponds to the term in the conjecture involving the square of the cubic derivatives of the potential with respect to the scalar field) should give a result at least as weak as the fourth-order interaction mediated by a four-dimensional graviton exchange, which is essentially the square of the second derivative of the potential with respect to the field (which defines the mass-squared of the scalar field). This is the philosophy behind (62). For noncritical strings with exponential potentials the situation needs to be revisited, which might lead to an explanation on the aforementioned discrepancy, and why the (noncanonically normalized) form $\Phi$ of the dilaton should be used in the string effective action.

Finally, we note that, for the dilaton-induced Starobinsky potential (54), in the inflationary phase occurring for large positive $\phi$, and with a canonical kinetic term as in (15), the condition (62) is trivially satisfied, as can be seen by direct computation. The exponent $\sqrt{2}$ is crucial for this result. In this model the potential (54) is of the following generic form for large $\kappa \phi \gg 1$ :

$$
V=\left|c_{5}\right|-\left|c_{6}\right| e^{-\sqrt{2} \kappa \phi}+\ldots
$$

with $c_{5}, c_{6} \in \mathbb{R}$ real constants, and with the kinetic term of $\phi$ canonically normalized. We see from (67) that the improved WGC (62) requires $4 \kappa^{6}<8 \kappa^{6}$, which is satisfied. We also remark that the condition is satisfied for this model 
also if one uses the noncanonically normalized standard dilaton $\Phi$ appearing in the string effective action (66) or (53).

However, we caution that there are many ambiguities surrounding the formulation of the WGC. For example, there are other forms of the WGC [34] that take into account the weakness of gravitational interactions, implying that the situation involving scalars and the WGC is still ambiguous, reflecting once more our ignorance of the structure of quantum gravity. Thus, although the conjecture that gravity must be the weakest force certainly sounds plausible for a quantum theory of gravity, the rigorous and unambiguous quantification of this conjecture is nevertheless not trivial. In particular, it is quite possible that the formulation of the scalar WGC (61) may need modification in the case of a noncritical string cosmology with a running dilaton, while maintaining the nature of gravity as the weakest observable force in our four-dimensional world. We conclude that there is no dramatic contradiction between the superstring cosmology scenarios we advocate and the weak gravity conjecture. Moreover, it seems clear from the discussion in Sec. III that these models are quite compatible with the swampland conjectures.

\section{ACKNOWLEDGMENTS}

The work of J. E. and N. E. M. is supported in part by the UK Science and Technology Facilities research Council (STFC) under the research Grant No. ST/P000258/1, and J.E. also receives support from an Estonian Research Council Mobilitas Pluss grant. The work of D. V.N. is supported in part by the DOE Grant No. DE-FG0213ER42020 at Texas A\&M University and in part by the Alexander S. Onassis Public Benefit Foundation. J. E. and N.E. M. participate in the COST Association Action CA18108 "Quantum Gravity Phenomenology in the Multimessenger Approach (QG-MM)". N. E. M. also acknowledges a scientific associateship ("Doctor Vinculado") at IFIC-CSIC, Valencia University, Valencia, Spain.
[1] H. Ooguri and C. Vafa, Nucl. Phys. B766, 21 (2007).

[2] G. Obied, H. Ooguri, L. Spodyneiko, and C. Vafa, arXiv:1806.08362.

[3] H. Ooguri, E. Palti, G. Shiu, and C. Vafa, Phys. Lett. B 788, 180 (2019).

[4] S. K. Garg and C. Krishnan, J. High Energy Phys. 11 (2019) 075.

[5] R. Brandenberger, V. Kamali, and R. O. Ramos, arXiv:2002 .04925 .

[6] F. Denef, A. Hebecker, and T. Wrase, Phys. Rev. D 98, 086004 (2018).

[7] J. R. Ellis, N. Mavromatos, and D. V. Nanopoulos, Phys. Lett. B 293, 37 (1992).

[8] J. R. Ellis, N. Mavromatos, and D. V. Nanopoulos, Mod. Phys. Lett. A 10, 1685 (1995).

[9] G. Diamandis, B. Georgalas, A. Lahanas, N. Mavromatos, and D. V. Nanopoulos, Phys. Lett. B 642, 179 (2006).

[10] J. R. Ellis, N. Mavromatos, and D. V. Nanopoulos, Chaos Solitons Fractals 10, 345 (1999).

[11] J. R. Ellis, N. Mavromatos, and D. V. Nanopoulos, Phys. Lett. B 619, 17 (2005); Gen. Relativ. Gravit. 37, 1665 (2005); G. Diamandis, B. Georgalas, N. Mavromatos, and E. Papantonopoulos, Int. J. Mod. Phys. A 17, 4567 (2002).

[12] S. Hellerman, N. Kaloper, and L. Susskind, J. High Energy Phys. 06 (2001) 003; W. Fischler, A. Kashani-Poor, R. McNees, and S. Paban, J. High Energy Phys. 07 (2001) 003; E. Witten, arXiv:hep-th/0106109; P. O. Mazur and E. Mottola, Phys. Rev. D 64, 104022 (2001).

[13] J. R. Ellis, N. Mavromatos, and D. V. Nanopoulos, arXiv: hep-th/0105206.

[14] I. Antoniadis, C. Bachas, J. R. Ellis, and D. V. Nanopoulos, Phys. Lett. B 211, 393 (1988); Nucl. Phys. B328, 117 (1989); Phys. Lett. B 257, 278 (1991).
[15] R. Bousso, J. High Energy Phys. 07 (1999) 004.

[16] G. Gibbons and S. Hawking, Phys. Rev. D 15, 2738 (1977).

[17] J. Ellis, N. E. Mavromatos, and D. V. Nanopoulos, Phys. Lett. B 732, 380 (2014).

[18] N. Aghanim et al. (Planck Collaboration), arXiv:1807 .06209 .

[19] E. Witten, Phys. Rev. D 44, 314 (1991); See also: Twodimensional string theory and black holes, in Quantum Gravity and Beyond, edited by M. Mansouri and J. J. Scanio (World Scientific Publishing Co., Ltd., Singapore, 1992), pp. 126-142, ISBN 981-02-1290-9.

[20] J. R. Ellis, N. Mavromatos, and D. V. Nanopoulos, Phys. Lett. B 278, 246 (1992).

[21] J. R. Ellis, N. Mavromatos, and D. V. Nanopoulos, Int. J. Mod. Phys. A 13, 1059 (1998); Int. J. Mod. Phys. A 12, 2639 (1997).

[22] J. R. Ellis, N. Mavromatos, and D. V. Nanopoulos, Gen. Relativ. Gravit. 32, 943 (2000).

[23] J. L. Lopez and D. V. Nanopoulos, Mod. Phys. Lett. A 11, 1 (1996).

[24] J. R. Ellis, N. E. Mavromatos, V. A. Mitsou, and D. V. Nanopoulos, Astropart. Phys. 27, 185 (2007); S. Basilakos, N. E. Mavromatos, V. A. Mitsou, and M. Plionis, Astropart. Phys. 36, 7 (2012).

[25] I. L. Shapiro and J. Solà, J. High Energy Phys. 02 (2002) 006; Phys. Lett. B 682, 105 (2009); J. Solà, J. Phys. A 41, 164066 (2008).

[26] S. Basilakos, N. E. Mavromatos, and J. Solà, Phys. Rev. D 101, 045001 (2020).

[27] G. Curci and G. Paffuti, Nucl. Phys. B286, 399 (1987). 
[28] G. A. Diamandis, B. C. Georgalas, N. E. Mavromatos, E. Papantonopoulos, and I. Pappa, Int. J. Mod. Phys. A 17, 2241 (2002).

[29] J. Alexandre, A. Kostouki, and N. E. Mavromatos, J. High Energy Phys. 03 (2009) 022.

[30] N. Arkani-Hamed, L. Motl, A. Nicolis, and C. Vafa, J. High Energy Phys. 06 (2007) 060.

[31] E. Palti, J. High Energy Phys. 08 (2017) 034.
[32] E. Gonzalo and L. E. Ibanez, J. High Energy Phys. 08 (2019) 118.

[33] A. Kusenko, V. Takhistov, M. Yamada, and M. Yamazaki, Phys. Lett. B 804, 135369 (2020),

[34] K. Benakli, C. Branchina, and G. Lafforgue-Marmet, arXiv:2004.12476; E. Gonzalo and L. E. Ibanez, arXiv: 2005.07720 . 